\title{
Clinical Tools for the Prevention and Treatment of Childhood and Adolescent PTSD
}

Rich Gilman, $P h D^{1,2,4, *}$

Jeffrey R. Strawn, $M D^{2,1}$

Brooks R. Keeshin, $M D^{3}$

\author{
Address \\ ${ }^{1}$ Cincinnati Children's Hospital Medical Center, Department of Pediatrics, Division \\ of Child and Adolescent Psychiatry, Cincinnati, $\mathrm{OH}$ 45219, USA \\ ${ }^{2}$ Department of Psychiatry and Behavioral Neuroscience, University of Cincinnati, \\ College of Medicine, Cincinnati, $\mathrm{OH}$ 45267, USA \\ ${ }^{3}$ Department of Pediatrics, University of Utah, Salt Lake, UT, USA \\ ${ }^{*}, 4$ Division of Child and Adolescent Psychiatry, Cincinnati Children's Hospital \\ Medical Center, 3333 Burnet Ave., MLC 3015, Cincinnati, OH 45229, USA \\ Email: richard.gilman@cchmc.org
}

Published online: 30 January 2015

(C) Springer International Publishing AG 2015

This article is part of the Topical Collection on Post-Traumatic Stress Disorders

Keywords Posttraumatic stress disorder (PTSD) - Children • Adolescents - Psychotherapy • Trauma-focused cognitive behavioral therapy (TF-CBT)

\section{Opinion Statement}

Posttraumatic stress disordxer (PTSD) represents a chronic and debilitating condition that affects the health and well-being of millions of youth. Traditional approaches focus on psychopharmacologic treatments and psychotherapeutic interventions. Although psychopharmacologic treatments are designed to address underlying physiological manifestations (e.g., hyperarousal, mood instability), they are less effective in modifying the underlying, distorted cognitions that maintain the disorder. Psychotherapeutic interventions are effective in alleviating individual symptoms, but their benefits do not extend to the larger social system that the youth is embedded. Given the importance of social support in treatment prognosis, addressing the larger family system is needed. The most effective approach to PTSD prevention and treatments thus emphasizes a biopsychosocial framework, such as an empirically informed algorithm that incorporates psychopharmacologic treatments, individual interventions, and family intervention approaches in a systematic fashion. 


\section{Introduction}

The prevalence of traumatic events among American youth is sobering. Recent studies report that as many as one quarter of all children in the USA witness or experience a traumatic event before age 4 ; nearly $14 \%$ repeatedly experience maltreatment by a caregiver (the majority of which will consist of neglect, physical abuse, or sexual abuse), and over $60 \%$ school-aged youth have or will experience a traumatic event in any given year $[1,2 \bullet$, $3]$. Equally alarming is that these rates are based on reported events; data obtained from community and school-based screenings indicate that the prevalence of traumatic events is considerably higher [4], making childhood trauma an insidious health risk [5].

Although the majority of traumatized youth recover, a substantial number develop posttraumatic disorder (PTSD) $[6,7]$. In recent years, a number of risk factors for the development of childhood PTSD have been identified, including: female gender, number of traumas experienced, greater exposure to the index trauma, presence of a preexisting psychiatric disorder, parental psychopathology, and availability of social support (particularly family) [8]. Whatever the risk factors, common symptoms associated with childhood PTSD include an involuntary intrusion of the traumatic event (such as distressing memories, flashbacks, repetitive play, and nightmares), persistent avoidance associated with the event, negative modifications of cognitions and mood, and marked alterations in arousal or reactivity to stimuli associated with the event [9]. Without adequate intervention, the long-term personal, social, and economic costs associated with childhood PTSD are staggeringly high. For example, adults with PTSD who experienced their first trauma before age 12 are more likely to develop severe depressive symptoms, to attempt suicide, to have relationship difficulties with their partners and/or children, to engage in substance abuse, and to be psychiatrically hospitalized [10]. Other studies examining psychiatric histories of youth in inpatient settings report that chronic trauma was a significant predictor of multiple axis I disorders, has higher likelihood of placement on medications (including antipsychotics), and has higher rates of obesity $[11,12]$.

Given the harmful effects associated with childhood PTSD, evidence-based interventions and treatments are urgently needed. Until recently, most evidence-based treatments focused on the symptoms within the affected individual while largely placing less consideration on the cascading effects of PTSD symptoms throughout the family system. In the following sections, we present data related to current pharmacotherapies as well as individual and family system approaches that address childhood PTSD. Our review is guided by an algorithm adapted from Keeshin and Strawn [13, 14]. As noted in the figure and as described below, we distinguish prevention from intervention approaches within these systems (Fig. 1).

\section{Psychopharmacologic approaches for youth with PTSD}

\section{Propranolol for the prevention of PTSD in youth}

- Several studies of traumatized adults suggest that the centrally acting, long-chain $\beta$-blocker, propranolol may prevent PTSD. However, this agent has only recently been examined in traumatized youth.

- Children and adolescents aged 10-18 years randomized to receive propranolol or placebo (within $12 \mathrm{~h}$ of accidental traumatization) did not exhibit differences in CAPS-CA scores or in the number of patients meeting criteria for PTSD or subthreshold criteria for PTSD at 6-week follow-up [15].

- Three studies have examined selective-serotonin reuptake inhibitors (SSRIs) in youth with PTSD and have failed to demonstrate 


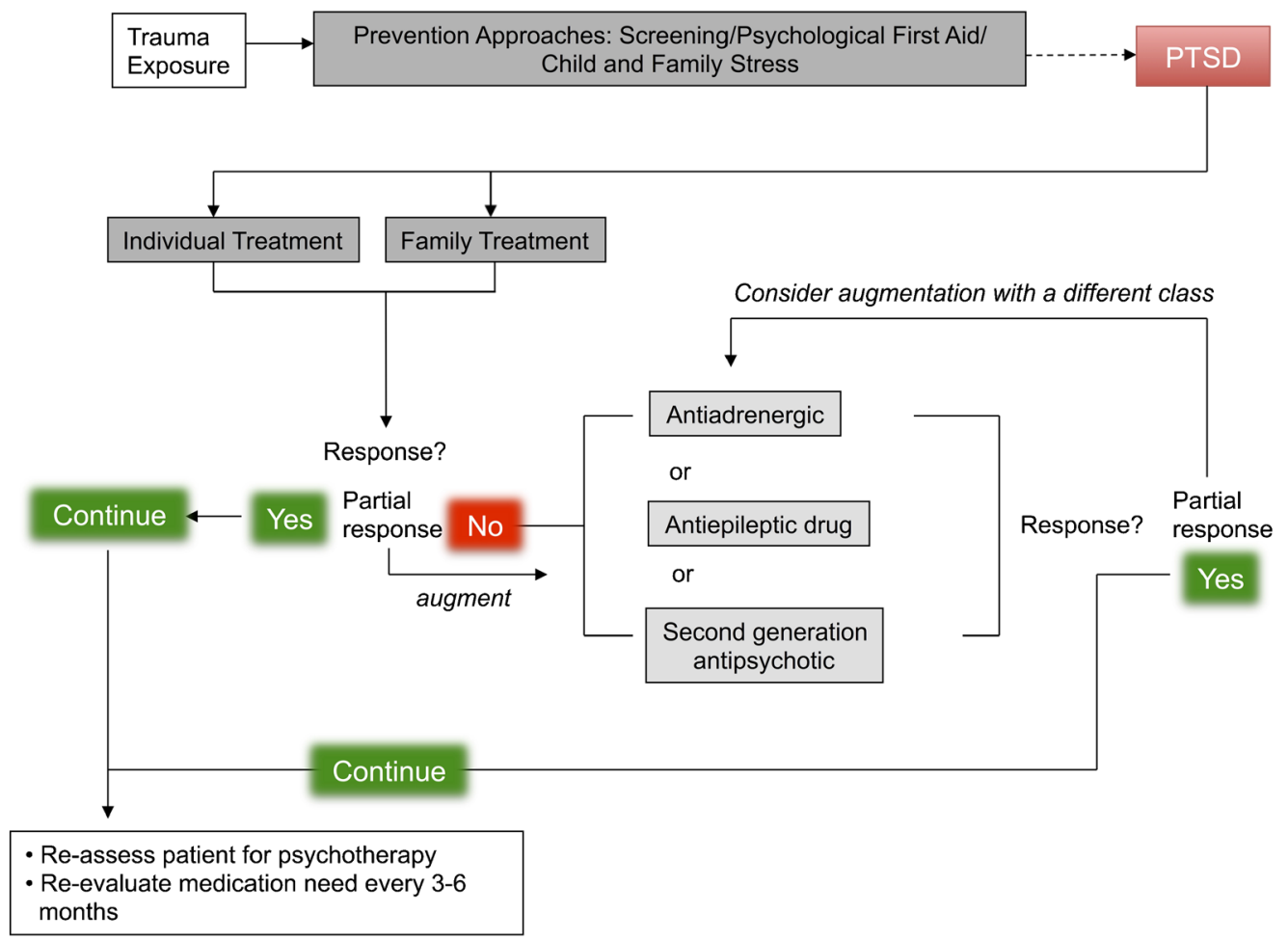

Proposed algorithm for the prevention and treatment of PTSD in children and adolescents. Of note, selective serotonin reuptake inhibitors may be most appropriate for patients with co-occurring anxiety disorders or major depressive disorder, although, they have shown little benefit in terms of core PTSD symptoms in youth. Psychotherapies are shown in dark gray boxes and psychopharmacologic interventions are shown in light gray boxes.

Fig. 1. Proposed algorithm for the prevention and treatment of PTSD in children and adolescents. Of note, selective serotonin reuptake inhibitors may be most appropriate for patients with co-occurring anxiety disorders or major depressive disorder, although, they have shown little benefit in terms of core PTSD symptoms in youth. Psychotherapies are shown in dark grey boxes, and psychopharmacologic interventions are shown in light gray boxes

significant advantages compared with placebo.

- Flexibly dosed sertraline (mean dose, $150 \mathrm{mg} /$ day; range, 50-200 mg/ day) vs. placebo (as adjunctive treatment) with trauma-focused cognitive behavioral therapy (TF-CBT) yielded only limited improvement in the sertraline-treated youth [16].

- Flexibly dosed sertraline, over the course of 10 weeks, did not result in significant differences (compared with placebo) in UCLA PTSD Scale scores (17-item total score), although it was noted to be generally well tolerated [17].

- Youth who were treated with fluoxetine or imipramine (compared with placebo) did not differ in acute stress disorder symptoms following acute thermal burn trauma; although, the duration of treatment was only 1 week [18] and thus it is unlikely that clinical benefit would have been observed.

- Finally, two open-label studies suggest improvement in PTSD symptoms in youth treated with citalopram [19, 20]. 
- Given that several studies suggest increased noradrenergic tone in both adults and youth with PTSD [14, 21], enthusiasm for antiadrenergic agents has increased.

- To date, multiple case reports suggest benefit for adjunctive prazosin $[22,23]$ and prazosin monotherapy $[24,25]$ in youth diagnosed with PTSD, although these initial encouraging reports have not been examined in large, double-blind, placebo-controlled trials.

- The $\alpha_{2}$-agonist clonidine appears to attenuate hyperarousal, hypervigilance, sleep disruption, exaggerated startle responses, and nightmares in open-label trials. In abused youth, clonidine decreases reenactment symptoms [26]. Similarly, the $\alpha_{2}$-agonist guanfacine may reduce nightmares in children with PTSD [27, 28].

- In children and adolescents with PTSD, extended-release guanfacine was examined with regard to the treatment of PTSD symptoms. In this study, improvements were noted for the UCLA Reaction Index scores for reexperiencing, avoidant, and hyperarousal symptoms [29].

Second-generation antipsychotics

- The efficacy of quetiapine has been examined in youth with PTSD and improvement in Traumatic Symptom Checklist for Children (TSCC) posttraumatic stress $t$-scores and in symptoms of anxiety, depression, and anger were noted over a 6-week treatment period, although significant weight gain was observed [30].

- Meighen and collegues [31] treated three preschool-aged children with serious thermal burns and acute stress disorder with risperidone and observed decreases in all symptom clusters of ASD [31]. More recently, Keeshin and Strawn [32] reported a significant improvement associated with adjunctive risperidone in a 13-year-old boy with severe sexual abuse- and neglectrelated PTSD.

- In one open-label trial of carbamazepine in pediatric patients with sexual abuse-related PTSD, carbamazepine treatment was associated with remission in the majority of patients and was well tolerated with no adverse events [33].

- In a separate open-label study, divalproex (either high or low dose) is also associated with improvements in CGI score over the course of treatment [34].

- A retrospective examination of patients (aged 6-14 years) who were treated with oxcarbazepine for mood symptoms and behavior problems associated with anger and irritability, moderate clinical global improvement was observed for most patients [35]. 
- Gupta and colleagues retrospectively evaluated the efficacy of adjunctive cyproheptadine in youth with PTSD and noted effects ranging from "complete remission to a decrease in the intensity and frequency of nightmares" [36].

- We are unable to locate any reports on the use of benzodiazepines in the management of PTSD in children and adults.

\section{Individual prevention approaches}

\section{Child adversity prevention}

- Prior childhood adversity is a significant predictor of subsequent childhood adversities and traumas [37].

- Analysis of the National Comorbidity Survey, a nationally representative sample, observed that childhood adversities were associated with nearly half of all childhood onset psychiatric disorders [38].

- Both physical and sexual abuse during childhood increase the likelihood of mood, anxiety, substance use, and disruptive behavior disorders later in life, accounting for anywhere from 50 to $100 \%$ increased risk of a specific cluster of psychiatric illnesses [38].

- Many child abuse prevention evidence-based models focus on the promotion of child well-being, reducing risk factors for abuse and enhancing skills and supports [39].

- Most often, child abuse prevention services are focused on families with younger children and may be delivered in the home, in the primary care setting, or through parent training programs.

- These programs measure success based on subsequent rates of recidivism or abuse.

- It is likely that by decreasing the childhood psychosocial stressors or adversities experienced within the family, a reduction in psychiatric morbidity may result from these and other child abuse prevention programs.

- It is important that any measure or assessment process include both an inquiry into histories of different types of adversity, as well as symptoms associated with those events.

- One example of such a measure is the UCLA PTSD RI for DSM 5, available in both parent and child/adolescent versions, which allows for the systematic evaluation of patients for adversity and trauma [40].

- The UCLA PTSD RI has four components, a timeline of prior trauma(s) that indicate the age at which the trauma(s) was experienced. A second component asks about specific traumatic events and ranks the events 


\section{Early assessments after trauma}

identified by the child as being the most traumatic. Third, 31 symptom questions assess the frequency at which DSM 5 PTSD criteria, including dissociative symptomatology, occur. Finally, the final part of the measure assesses for functional impairment.

- Utilizing a standardized measure allows for a complete understanding of the range and frequency of symptoms, and with repeated use is a nice measure to support change in illness over time.

\section{Psychological first aid}

- Psychological first aid (PFA) is a systemic, integrated approach to address the acute mental health needs of a community or population after disaster or other large-scale traumas [41].

- The primary components of psychological first aid include (1) responding to or initiating contact with those exposed to the trauma, (2) enhancing safety and providing physical and emotional comfort for survivors, (3) identifying and providing additional support to overwhelmed survivors, (4) identifying current needs, (5) offering practical assistance for immediate needs, (6) re-establishing connections with support systems, (7) providing psychoeducation on normal responses to stress and effective coping strategies, and (8) linking survivors to available services.

\section{Individual intervention approaches}

\section{Psychological treatment overview}

- Psychotherapeutic treatments demonstrate effectiveness in diminishing the impacts of abuse and other forms of trauma.

- Many of the currently used psychotherapies for the treatment of trauma incorporate both exposure and cognitive processing into manualized treatments.

- Exposure components incorporate new information into an existing memory structure to reduce or eliminate fear and anxiety associated with a current stimulus, leading to a corrective emotional experience.

- Cognitive processing addresses and corrects negative cognitive schemas and cognitive distortions that frequently occur after trauma, resulting in the formation of a more healthy incorporation of traumatic experiences into one's self-identity and view of the world.

- For traumatized children, cognitive behavioral-based treatments demonstrated a Cohen's $d$ effect size of 0.50 in the reduction of symptoms of posttraumatic stress when compared with controls [42]. 
- For sexually abused children, effect size of 1.41 for cognitive behavioral therapies for the reduction of psychological distress and effect sizes of $0.71,1.39$, and 2.18 for the reduction of PTSD/trauma symptoms at 1$3,4-6$, and $>6$ months follow-up have been demonstrated [43].

- CBT basis, structured and sequential format, individual treatment, family involvement, and length of treatment are factors consistently associated with increased effect size in the treatment of childhood PTSD [44•].

- Non-CBT-based individual therapies such as Child Parent Psychotherapy and Eye Movement Desensitization and Reprocessing target PTSD symptoms in children and have positive findings in the literature but less empirical support.

- Meta-analysis of non-CBT-based treatments demonstrates smaller effects in the reduction of posttraumatic stress symptoms, with Cohen's $d$ effect size of 0.19 [44•].

- Various treatments appear more effective for some sequelae than others, and careful assessment of the effects of abuse or other types of trauma on individual children is the best way to decide which specific treatments are most likely to help that child.

\section{Trauma-focused cognitive behavioral therapy}

- Trauma-focused cognitive behavioral therapy (TF-CBT) is a manualized, short, 12-16-week individual treatment for sexual abuse and other forms of trauma in ages 3-17 [45].

- TF-CBT is a stepwise approach for the treatment of individuals who have been traumatized, either acutely or chronically, and suffer psychiatric and/or behavioral complications from their trauma.

- Patients who benefit from TF-CBT include those with PTSD, anxiety, depressive, or behavior and self-image changes that resulted from a traumatic event or series of traumas.

- The therapy consists of psychoeducational, cognitive and stress reduction components, and the development of a trauma narrative.

- The use of TF-CBT as an evidence-based treatment in children exposed to trauma is supported by multiple randomized controlled trials comparing TF-CBT efficacy against other psychotherapies, and trials have been performed by multiple investigators who have replicated findings of the original treatment developers [14].

\section{TF-CBT components}

- Psychoeducation is initially provided to patients to help them understand trauma symptoms and informs the child and family about the normalcy of their reactions to the traumatic experience.

- Prior to cognitive processing and controlled exposure to the traumatic memories, the therapist teaches the child self-relaxation techniques 


\section{Prolonged exposure therapy for adolescents}

- Manualized 12-15-session approach, effective for youth aged 12 to 18 years.

- Prolonged exposure therapy for adolescents (PE-A) consisting of three main components: (1) psychoeducation, treatment planning, and the teaching of self-relaxation techniques such as breathing exercises, (2) development of an exposure hierarchy, practiced in vivo exposure during sessions and assigning in vivo exposures such as listening to taped recordings by the patient recounting the trauma, and (3) termination and relapse prevention, including addressing potential future challenges.

- Compared with a manualized, conflict-based, psychodynamic intervention, adolescents who received PE-A demonstrated a greater reduction in symptoms of posttraumatic stress and improved rate of PTSD remission both at the end of treatment and at follow-up [46]. Limitations of the study include differences in the frequency of treatment between the groups as well as experience of the therapists.

\section{Family system PTSD prevention approaches}

\section{Child and family stress intervention}

- Developed as a secondary prevention to address early distressing symptoms of a severe psychiatric disorder (including PTSD).

- Best suited for recently traumatized children with new onset of symptoms or worsening symptoms shortly after the trauma.

- Involves four to six sessions for youth (ages 7-17) and their adult caregivers. 
- Focuses on three key risk factors that contribute to the development of PTSD: poor communication within the family, inability to identify trauma symptoms, and inadequate coping mechanisms.

- Target symptoms include anxiety, depression/withdrawal, intrusive thoughts, sleep disturbances, externalizing, and sexualized behaviors.

- The caregiver attends the first session to receive psychoeducation and provide their observations of the child's symptoms; the child discusses her symptoms for the first part of the second session, and the second portion of the second session with caregiver and child present focuses on improving communication and identifying most significant symptoms; the parent and child together attend all subsequent sessions to review the utility of coping skills, reduction of symptoms, and parentchild communication.

- A recent study conducted by Berkowtiz, Stover, and Marans [47] randomly assigned youth $(N=106$, ages $7-17$ years) to child and family stress intervention (CFTSI) or a supportive comparison group and observed that CFTSI-treated patients were $65 \%$ less likely to meet criteria for PTSD at 3-month follow-up than youth in the comparison group. CFTSI was also associated with lower levels of behavior avoidance and re-experiencing of the event (there were no differences with respect to hyperarousal).

- Viewed as a natural bridge to evidence-based therapies such as TF-CBT in children who need more comprehensive trauma treatment after the initial four to six sessions.

\section{Family system PTSD intervention approaches}

\section{Family-focused cognitive therapy and cognitive behavioral therapy (FFCBT)}

- Incorporates components of TF-CBT to involve the affected child and his/her caregivers.

- Components of family-focused cognitive therapy and cognitive behavioral therapy (FFCBT) include psychoeducation, relaxation training and anxiety management, cognitive restructuring, exposure therapy for the affected child, and therapeutic efforts to improve communication, problem solving, and behavior management skills for parents. Components of family-focused cognitive therapy (FFCT) are the same but exclude exposure.

- Published studies are based on ten weekly sessions, approximately $1 \mathrm{~h}$ in length $[48,49]$.

- A recent study compared youth experiencing single-incident trauma (and subsequently diagnosed with PTSD, $N=33$ ), in which patients, following randomized to either FFCT or FFCBT and following treatment, did not differ in PTSD symptoms and problematic behaviors. However, FFCBTtreated patients reported lower anxiety than those who were treated with FFCT. These effects were noted at 6 months posttreatment [49]. 
Risk reduction through family therapy

- Builds upon individualized approaches that target PTSD, comorbid substance abuse, and other negative sequelae-risk reduction through family therapy (RRFT) [50].

- Involves the affected youth and his or her adult caregivers and focuses on seven treatment components: psychoeducation, coping, family communication, substance abuse, PTSD, healthy dating and sexual decision-making, and re-victimization risk reduction.

- The therapy consists of approximately 34 weekly sessions that are held for 60-90 min.

- In a recent randomized controlled trial of 30 treatment-seeking adolescents of sexual abuse, half of the sample received RRFT while the other half received treatment as usual. RRFT-treated adolescents reported greater reductions in PTSD, substance use, and internalizing symptoms than (comparison group). Furthermore, adolescents and their caregivers reported increased family cohesion, which is a necessary component of positive prognosis. Effect size differences were noted at 6-month posttreatment.

\section{Cognitive behavioral intervention for trauma in schools-family treatment}

- Expands on school-based trauma-focused interventions that incorporate psychoeducation, relaxation training, cognitive therapy, trauma exposure, and social problem solving to address trauma in the affected child.

- Cognitive behavioral intervention for trauma in schools-family treatment (CBITS + family) also includes parent-specific curricula designed to promote effective parent-child communication, family cohesion, stress management, and behavior management skills.

- Sessions are between 10 and 13 weeks for the child and four parentspecific sessions. Length of sessions are between 1 and $2 \mathrm{~h}$.

- A recent pilot study conducted by Santiago [51] examined youth assigned to the CBITS as usual or CBITS + family condition. In this quasiexperimental study, significant greater reductions in symptom severity and greater improvements in positive mental health indicators were found among youth in the CBITS + family condition. In addition, parents in this condition reported significantly greater attitudes towards mental health, better coping strategies, and higher levels of parenting consistency.

\section{Conclusion}

The impact of child PTSD does not only affect intra-individual functioning but also ripples across the entire family system. Our review, based on the algorithm in the figure, represents the next wave of treatments that combine psychopharmacological, individual, and family approaches into a unified framework. Clearly, additional studies are needed to empirically assess the efficacy of this 
framework, but clinicians may find value in considering the framework into their own practice using the empirically based treatments described herein.

\section{Compliance with Ethics Guidelines}

Conflict of Interest

Dr. Gilman has received research funding from the Eunice Kennedy Shriver National Center for Child Health Development.

Dr. Strawn has received research support from Eli Lilly, Forest Research Institute, Lundbeck, Shire, the American Academy of Child and Adolescent Psychiatry, and the National Institute of Mental Health.

Dr. Keeshin reports no conflicts.

\section{Human and Animal Rights and Informed Consent}

This article includes studies with human subjects performed by the authors. All studies had Institutional Review Board approval, and Informed Consent was obtained from all participants.

\section{References and Recommended Reading}

Papers of particular interest, published recently, have been highlighted as:

- Of importance

1. National Center for Mental Health Promotion and Youth Violence Prevention. Childhood trauma and its effect on healthy development. (2012)

2.• Finkelhor D, Turner HA, Shattuck A, Hamby SL. Violence, crime, and abuse exposure in a national sample of children and youth: an update. JAMA Pediatr. 2013;167(7):614-21. doi:10.1001/jamapediatrics. 2013.422013.

This article provides a current update on the prevalence of traumatic experiences in youth.

3. Finkelhor D, Turner H, Ormrod R, Hamby SL. Violence, abuse, and crime exposure in a national sample of children and youth. Pediatrics. 2009;124(5):141123. doi:10.1001/jamapediatrics.2013.42.

4. Horowitz K, McKay M, Marshall R. Community violence and urban families: experiences, effects, and directions for intervention. Am J Orthopsychiatr. 2005;75(3):356-68. doi:10.1037/0002-9432.75.3. 356.

5. Lanius RA, Vermetten E, Pain C. The impact of early life trauma on health and disease: the hidden epidemic. Cambridge: Cambridge University Press; 2010.

6. Blom M, Oberink R. The validity of the DSM-IV PTSD criteria in children and adolescents: a review. Clin Child Psychol Psychiatr. 2012;17(4):571-601. doi:10. 1177/1359104511426408.
7. Kessler RC, Avenevoli S, Costello J, et al. Severity of 12month DSM-IV disorders in the National Comorbidity Survey Replication Adolescent Supplement. Arch Gen Psychiatr. 2012;69(4):381-9. doi:10.1001/ archgenpsychiatry.2011.1603.

8. Gilman R, Chard KM, Holleb L, Teeters A. Understanding and managing behaviors of children diagnosed with posttraumatic stress disorder (PTSD). In: Kolbert JB, Crothers LM, editors. Understanding and managing behaviors of children with psychological disorders: A reference for classroom teachers. New York: Bloomsbury Press, 267-284

9. American Psychiatric Association. Diagnostic and Statistical Manual of Mental Disorders. 5th ed. Arlington: American Psychiatric Association; 2013.

10. American Academy of Child and Adolescent Psychiatry. Practice parameter for the assessment and treatment of children and adolescents with posttraumatic stress disorder. J Am Acad Child Adolesc Psychiatr. 2010;49(4):414-30.

11. Keeshin RB, Strawn JR, Luebbe AM, Saldana SN, Wehry AM, DelBello MP, et al. Hospitalized youth and child abuse: a systematic examination of psychiatric morbidity and clinical severity. Child Abuse Negl. 2014;38(1):76-83. doi:10.1016/j. chiabu.2013.08.013. 
12. Keeshin BR, Luebbe AM, Strawn JR, Saldana SN, Wehry AM, DelBello MP. Sexual abuse is associated with obese children and adolescents admitted for psychiatric hospitalization. J Pediatr. 2013;163(1):154-9. doi:10.1016/j.jpeds.2012.12.091.

13. Foa EB, Keane TM, Friedman MJ, Cohen JA. Effective treatments for PTSD: Practice guidelines from the International Society of Traumatic Stress Studies 2nd ed. New York: The Guilford Press

14. Keeshin BR, Strawn JR. Psychological and pharmacologic treatment of youth with posttraumatic stress disorder: an evidence-based review. Child Adolesc Psychiatric Clin N Am. 2014;23:399-411.

15. Nugent NR, Christopher NC, Crow JP, Browne L, Ostrowski S, Delahanty DL. The efficacy of early propranolol administration at reducing PTSD symptoms in pediatric injury patients: a pilot study. J Trauma Stress. 2010;23(2):282-7. doi:10.1002/jts.20517.

16. Cohen JA, Mannarino AP, Perel JM, Staron V. A pilot randomized controlled trial of combined traumafocused CBT and sertraline for childhood PTSD symptoms. J Am Acad Child Adolesc Psychiatr.

2007;46(7):811-9. doi:10.1097/chi. ob013e3180547105.

17. Robb AS, Cueva JE, Sporn J, Yang R, Vanderburg DG. Sertraline treatment of children and adolescents with posttraumatic stress disorder: a double-blind, placebocontrolled trial. J Child Adolesc Psychopharmacol. 2010;20(6):463-71. doi:10.1089/cap.2009.0115.

18. Robert R, Tcheung WJ, Rosenberg L, Rosenberg M, Mitchell C, Villarreal C, et al. Treating thermally injured children suffering symptoms of acute stress with imipramine and fluoxetine: a randomized, double-blind study. Burns. 2008;34(7):919-28.

19. Seedat S, Stein DJ, Ziervogel C, Middleton T, Kaminer D, Emsley RA, et al. Comparison of response to a selective serotonin reuptake inhibitor in children, adolescents, and adults with posttraumatic stress disorder. J Child Adolesc Psychopharmacol. 2002;12(1):37-46.

20. Seedat S, Lockhat R, Kaminer D, Zungu-Dirwayi N, Stein DJ. An open trial of citalopram in adolescents with post-traumatic stress disorder. Int Clin Psychopharmacol. 2001;16(1):21-5.

21. Strawn JR, Geracioti Jr TD. Noradrenergic dysfunction and the psychopharmacology of posttraumatic stress disorder. Depress Anxiety. 2008;25(3):260-71.

22. Brkanac Z, Pastor JF, Storck M. Prazosin in PTSD. J Am Acad Child Adolesc Psychiatr. 2003;42(4):384-5.

23. Oluwabusi OO, Sedky K, Bennett DS. Prazosin treatment of nightmares and sleep disturbances associated with posttraumatic stress disorder: two adolescent cases. J Child Adolesc Psychopharmacol. 2012;22(5):399-402.Strawn et al., 2009.

24. Strawn JR, Delbello MP, Geracioti TD. Prazosin treatment of an adolescent with posttraumatic stress disorder. J Child Adolesc Psychopharmacol. 2009;19(5):599-600.
25. Strawn JR, Keeshin BR. Successful treatment of posttraumatic stress disorder with prazosin in a young child. Ann Pharmacother. 2011;45(12):1590-1.Porter and Bell, 1999.

26. Harmon RJ, Riggs PD. Clonidine for posttraumatic stress disorder in preschool children. J Am Acad Child Adolesc Psychiatr. 1996;35(9):1247-9.

27. Horrigan JP, Barnhill LJ. The suppression of nightmares with guanfacine. J Clin Psychiatr. 1996;57(8):371.

28. Horrigan JP. Guanfacine for PTSD nightmares. J Am Acad Child Adolesc Psychiatr. 1996;35(8):975-6.

29. Connor DF, Grasso DJ, Slivinsky MD, Pearson GS, Banga A. An open-label study of guanfacine extended release for traumatic stress related symptoms in children and adolescents. J Child Adolesc Psychopharmacol. 2013;23(4):244-51.

30. Stathis S, Martin G, McKenna JG. A preliminary case series on the use of quetiapine for posttraumatic stress disorder in juveniles within a youth detention center. J Clin Psychopharmacol. 2005;25(6):539-44.

31. Meighen KG, Hines LA, Lagges AM. Risperidone treatment of preschool children with thermal burns and acute stress disorder. J Child Adolesc Psychopharmacol. 2007;17(2):223-32.

32. Keeshin BR, Strawn JR. Risperidone treatment of an adolescent with severe posttraumatic stress disorder. Ann Pharmacother. 2009;43(7):1374.

33. Looff D, Grimley P, Kuller F, Martin A, Shonfield L. Carbamazepine for PTSD. J Am Acad Child Adolesc Psychiatr. 1995;34(6):703-4.

34. Steiner H, Saxena KS, Carrion V, Khanzode LA, Silverman M, Chang K. Divalproex sodium for the treatment of PTSD and conduct disordered youth: a pilot randomized controlled clinical trial. Child Psychiatry Hum Dev. 2007;38(3):183-93.

35. Staller JA, Kunwar A, Simionescu M. Oxcarbazepine in the treatment of child psychiatric disorders: a retrospective chart review. J Child Adolesc Psychopharmacol. 2005;15(6):964-9.

36. Gupta S, Austin R, Cali LA, Bhatara V. Nightmares treated with cyproheptadine. J Am Acad Child Adolesc Psychiatr. 1998;37(6):570-2.

37. Dong M, Anda RF, Felitti VJ, Dube SR, Williamson DF, Thompson TJ, et al. The interrelatedness of multiple forms of childhood abuse, neglect, and household dysfunction. Child Abuse Negl. 2004;28:771-84.

38. Green JG, McLaughlin KA, Berglund PA, Gruber MJ, Sampson NA, Zaslavsky AM, et al. Childhood adversities and adult psychiatric disorders in the national comorbidity survey replication I: associations with first onset of DSM-IV disorders. Arch Gen Psychiatr. 2010;67:113-23. doi:10.1001/archgenpsychiatry. 2009.186.

39. Krugman SD, Lane WG, Walsh CM. Update on child abuse prevention. Curr Opin Pediatr. 2007;19:711-8.

40. Pynoos RS, Steinberg AM. UCLA PTSD Reaction Index for Children and Adolescents-DSM 5 (2013). 
41. National Child Traumatic Stress Network and National Center for PTSD, Psychological First Aid: Field Operations Guide (2005).

42. Silverman WK, Ortiz CD, Viswesvaran C, Burns BJ, Kolko DJ, Putnam FW, et al. Evidence-based psychosocial treatments for children and adolescents exposed to traumatic events. J Clin Child Adolesc Psychol. 2008;37:156-83. doi:10.1080/15374410701818293.

43. Hetzel-Riggin MD, Brausch AM, Montgomery BS. A meta-analytic investigation of therapy modality outcomes for sexually abused children and adolescents: an exploratory study. Child Abuse Negl. 2007;31:125-41.

44. Harvey ST, Taylor JE. A meta-analysis of the effects of psychotherapy with sexually abused children and adolescents. Clin Psychol Rev. 2010;30:517-35. doi:10. 1016/j.cpr.2010.03.006.

This article provides a good review of the effects of individual treatments for youth suffering from sex abuse.

45. Cohen JA, Kelleher KJ, Mannarino AP. Identifying, treating, and referring traumatized children: the role of pediatric providers. Arch Pediatr Adolesc Med. 2008;162:447-52. doi:10.1001/archpedi.162.5.447.

46. Foa EB, McLean CP, Capaldi S, Rosenfield D.

Prolonged exposure vs supportive counseling for sexual abuse-related PTSD in adolescent girls: a randomized clinical trial. JAMA. 2013;310:2650-7. doi:10. 1001/jama.2013.282829.
47. Berkowitz SJ, Stover CS, Marans SR. The child and family traumatic stress intervention: secondary prevention for youth at risk of developing PTSD. J Child Psychol Psychiatr. 2011;52(6):676-85. doi:10.1111/j. 1469-7610.2010.02321.x.

48. Cobham VE, Dadds MR, Spence SH, McDermott B. Parental anxiety in the treatment of childhood anxiety: a different story three years later. J Clin Child Adolesc Psychol. 2010;39(3):410-20. doi:10.1080/ 15374411003691719.

49. Nixon RD, Sterk J, Pearce A. A randomized trial of cognitive behaviour therapy and cognitive therapy for children with posttraumatic stress disorder following single-incident trauma. J Abnorm Child Psychol. 2012;40(3):327-37. doi:10.1007/s10802-011-9566-7.

50. Danielson CK, McCart MR, Walsh K, de Arellano MA, White D, Resnick HS. Reducing substance use risk and mental health problems among sexually assaulted adolescents: a pilot randomized controlled trial. J Fam Psychol. 2012;26(4):628-35. doi:10.1037/a0028862.

51. Santiago CD, Lennon JM, Fuller AK, Brewer SK, Kataoka SH. Examining the impact of a family treatment component for CBITS: when and for whom is It helpful? J Fam Psychol. 2014;28(4):560-70. doi:10. 1037/a0037329. 